\title{
REVISIÓN CRÍTICA DE LOS ALCANCES Y LÍMITES DE LA GESTIÓN INTEGRADA DE RECURSOS HÍDRICOS (GIRH): EL CASO DE LA SUBCUENCA SANTA EULALIA ${ }^{1}$
}

\author{
Víctor Guevara Floríndez \\ Investigador independiente
}

\begin{abstract}
Resumen: La subcuenca Santa Eulalia es esencial para la ciudad de Lima pues provee agua para su población, la cual equivale casi a la tercera parte de la población nacional. Este artículo pone de relieve las expectativas e intereses en torno al agua, tanto por los actores locales de la subcuenca como por los que están fuera de ella, como la ciudad de Lima.

La nueva Ley de Recursos Hídricos (2009) y las normas posteriores pretendieron superar el enfoque tradicional de la administración del agua como recurso, pasando a una gestión integrada que, entre otras cosas, considere las dimensiones social, económica y ambiental del agua y aplique un enfoque participativo y de cuenca. Sin embargo, pasar de la promesa a la realidad es un gran reto.

En la primera sección del artículo presento un contexto general de la subcuenca, sus actores y su relación con la ciudad de Lima en el marco de las relaciones entre el campo y la ciudad. En la segunda explico la dinámica entre los usuarios de agua de la Subcuenca y de la ciudad de Lima, señalando algunos de los conflictos por el recurso y las alternativas que se vienen desarrollando para la gobernanza del agua. En la tercera sección reviso literatura crítica sobre la GIRH, incluidas las críticas al desarrollo y los estudios sobre la paz. Ello conduce a pensar que la GIRH como está siendo implementada es insuficiente para integrar las necesidades y visiones locales sobre el agua.
\end{abstract}

Palabras clave: GIRH, gestión del agua, gobernanza del agua, transformación de conflictos, desarrollo, postdesarrollo, cuenca, subcuenca, Santa Eulalia.

1 El artículo tiene como base la tesis del autor para obtener el grado de Maestría del Programa de Paz, Desarrollo, Seguridad y Transformación de Conflictos Internacionales de la Universidad de Innsbruck. 


\title{
Critical review of the scope and limits of Integrated Water Resources Management (IWRM): the case of the Santa Eulalia sub-basin
}

\begin{abstract}
The Santa Eulalia Sub-basin is essential for Lima city. It provides water for its population, which is almost the third part of the national population. This article focus on the expectation and interests on water of the actors of the Sub-basin, as well as the ones beyond it, like Lima city.

The new Water Law (2009) and the subsequent norms pretended to overtake the traditional approach to managing water as a resource to turn to an integrated management that, among other things, considers social, economic and environmental dimensions and apply a participative basin-based approach. Nonetheless, go from the promise to reality represents a big challenge.

The first section of the article presents a general context of the Subbasin, its actors and its relation to Lima city in the framework of relations between urban and rural areas. The second section explains the dynamics between the water users in the Sub-basin and Lima city, pointing out some of the conflicts for the resource and the alternatives being developed for water governance. In the third section, I present a critical review of IWRM, including critiques to development and peace studies. This leads to think that IWRM as is being implemented is insufficient to integrating local needs and visions on water.
\end{abstract}

Keywords: IWRM, water management, water governance, conflict transformation, development, post-development, basin, sub-basin, Santa Eulalia.

\section{Víctor Guevara Floríndez}

Master en Paz, Desarrollo, Seguridad y Transformación de Conflictos Internacionales por la Universidad de Innsbruck, Austria. Master en Recursos Naturales y Paz por la Universidad para la Paz de Naciones Unidas, Costa Rica. Posgrado en Gestión Integrada de los Recursos Hídricos por la PUCP. Licenciado en Derecho por la Facultad de Derecho de la Universidad de Lima. Especialista en temas de derecho ambiental. Actualmente forma parte del staff de asesores externos de TYTL Abogados.

Correo electrónico: victorguev@gmail.com 


\section{Introducción}

La subcuenca Santa Eulalia tiene gran importancia y merece aún mayores esfuerzos de los realizados hasta ahora para lograr el balance entre su conservación y el uso sostenible del agua.

Por un lado, esta subcuenca provee el agua y la energía - por generación hidroeléctrica- para la ciudad de Lima, que alberga nada menos que la tercera parte de la población del país: un uso inadecuado o la falta de decisiones sobre el futuro podrían afectar gravemente la sostenibilidad de la ciudad.

De otro lado, a diferencia de otras cuencas donde se han venido implementando Consejos de Recursos Hídricos de Cuenca (CRHC) en ámbitos más extensos definidos por la autoridad bajo criterios técnico-normativos, en Santa Eulalia se han venido desarrollando iniciativas con participación y coordinación entre usuarios locales, como el grupo de trabajo del Programa Agua, Clima y Desarrollo (PACyD). Esto, además de ser anterior a la conformación del Consejo de Recursos Hídricos de Cuenca Chillón - Rímac - Lurín (CRHC CHIRILU), representa un esfuerzo de articulación a un nivel menor que el del Consejo. En ese sentido, es un caso de estudio que puede ser demostrativo de qué hacer en otros Consejos que justamente adolecen de articulación con los niveles menores de cuenca.

Finalmente, puede graficar la toma de decisiones no solo con escasa participación local, sino además con miras a resolver necesidades de actores de fuera de la subcuenca, vale decir de la ciudad de Lima, con actores muy próximos al Gobierno central.

\section{Contexto de la subcuenca Santa Eulalia en relación a Lima}

El río Santa Eulalia es el principal afluente del río Rímac que, por su parte, constituye la mayor fuente de agua para la población de Lima y para la empresa Sedapal, que presta el servicio. No obstante, como veremos, gran parte de su caudal proviene de la cuenca del Mantaro y es traída mediante trasvases del otro lado de la cordillera. 
La subcuenca del río Santa Eulalia se ubica en la Provincia de Huarochirí y abarca diez distritos: Callahuanca, Carampoma, Huachupampa, Huanza, San Juan de Iris, San Pedro de Casta, San Pedro de Laraos, Santa Eulalia, San Mateo de Otao, San Antonio, aunque los dos últimos solo están parcialmente en la subcuenca.

A pesar de su cercanía a la ciudad de Lima (aproximadamente 50 $\mathrm{km}$ ), el distrito de Santa Eulalia, ubicado en la parte baja de la subcuenca del mismo nombre, adolece de ciertos problemas de conectividad con el resto de la subcuenca. De Santa Eulalia a los centros poblados de la parte alta de la subcuenca, puede haber rutas que duran hasta más de tres horas en auto. No obstante, el transporte público entre estas zonas es más bien esporádico y de baja calidad, por la baja densidad poblacional. Algo parecido ocurre con el acceso a una serie de servicios básicos que son cada vez menores, según se alejan de la ciudad de Lima.

Además de Sedapal, la subcuenca alberga empresas generadoras que proveen la mayor parte de energía para Lima, así como para otras partes del país. Por ende, el mayor número de interesados en la subcuenca está fuera de su ámbito, en los millones de usuarios que se benefician de dichos servicios en la ciudad de Lima. Así, no sorprende que muchos actores públicos y privados valoren la subcuenca como proveedora de recursos.

Conviene recordar el contexto histórico de las migraciones para entender mejor las relaciones que hay entre la subcuenca y Lima por el agua, que, al fin y al cabo, muestran en parte las relaciones entre el campo y la ciudad. En efecto, durante el siglo XX las grandes migraciones del campo a la ciudad cambiaron la configuración social del país. De hecho, «en los últimos setenta años la población urbana del Perú creció de un 35\% del total de población en 1940 a un 76\% en el año 2007» (Instituto de Planificación de Paisaje y Ecología 2014: 29). Ya a fines del siglo pasado, Matos Mar (1984:72) señalaba que de 1940 a 1984, Lima aumentó su población casi diez veces, «acompañando al gran cambio del país que en 1940 era rural (65\%) y ahora urbano (65\%)».

Además, el autor explicó que tan solo en parte del siglo XX la superficie urbana de Lima se incrementó drásticamente: de 3.166 hectáreas en 1920 pasaron a 3.966 hectáreas (800 nuevas hectáreas) para 1940. E incrementaron otras 4.711 de 1940 a 1961. Mientras en los siguientes veinte años hubo un crecimiento explosivo de 19.716 hectáreas.

De la población inmigrante que afluyó de los 24 departamentos del país, correspondía la más alta proporción a Ancash (10,6\%), Ayacucho (8.38\%), Junín (8,11\%) y la más baja a Madre de Dios con 
REVISIÓN CRÍTICA DE LOS ALCANCES Y LÍMITES DE LA GESTIÓN INTEGRADA DE RECURSOS HÍDRICOS (GIRH): El CASO de la Subcuenca Santa Eulalia / Víctor Guevara

provenían de las otras provincias del departamento de Lima, especialmente de distritos serranos ${ }^{2}$ (Matos Mar 1984: 73).

El abandono de las áreas ocupadas en las partes altas de las cuencas produjo la devaluación y reducción de tierras agrícolas, mientras en las partes bajas el aumento de la población se produjo mediante una ocupación carente de consideraciones ambientales o de seguridad, habitando los márgenes de la ciudad sin que las autoridades hubieran podido planificarlo. Esto ocasionó, con el tiempo, descuido en las partes altas de la cuenca, así como mayor presión sobre el recurso agua en la parte baja (la ciudad) para abastecer del servicio a la población.

La migración y los cambios sociales y ambientales que ella generó no son casuales. Mientras mayor es la cercanía a Lima, mayores oportunidades sociales. Si comparamos las condiciones de la población, según PNUD, al $2012^{3}$ Lima Metropolitana tenía un índice de desarrollo humano (IDH) de 0.6420 y los ciudadanos una esperanza de vida al nacer de 79,41 años. El $82,44 \%$ de su población tenía secundaria completa y el ingreso familiar per cápita era de S/ 1.026. La provincia de Huarochirí — donde está la subcuenca Santa Eulalia - tenía un IDH de 0,4742, una esperanza de vida de 72,76 años, un $70,87 \%$ de la población con secundaria completa y un ingreso familiar per cápita de S/ 572,3.

Dentro de la subcuenca la relación es semejante. El distrito de Santa Eulalia (en la parte baja) tenía en 2012 un IDH de 0,5697, bastante mejor al promedio de la provincia de Huarochirí, y un ingreso per cápita de S/ 906,4; mientras el distrito de Huanza, en la parte alta de la subcuenca, mostraba un IDH de 0,2621 y un ingreso per cápita de S/ 128.

\footnotetext{
2 Texto en negrita resaltadas por el autor.

3 Información recabada del Índice de Desarrollo Humano departamental, provincial y distrital del PNUD Perú.
} 


\section{Mapa 1 \\ Distribución de los distritos en las tres zonas de la subcuenca Santa Eulalia}

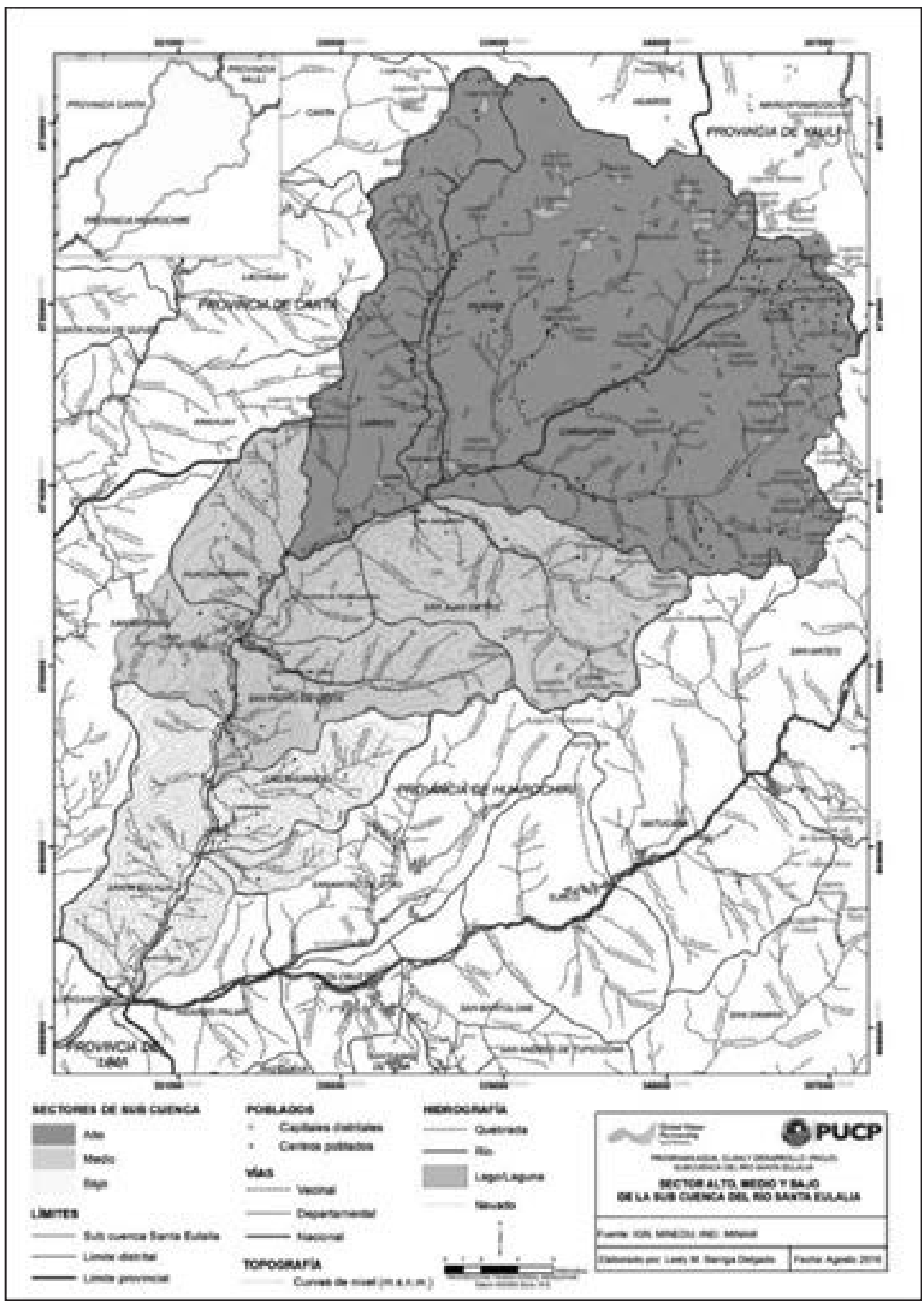

Fuente y elaboración: García Rivero (2017). 
Las diferencias tienen relación con las alternativas económicas que tienen en cada zona. En promedio, en la Provincia de Huarochirí predomina la actividad agrícola, con el 35,71\% de la PEA ocupada dedicada a ella, aunque en Santa Eulalia la principal actividad es transportes y comunicaciones, con 14,99\% de la PEA ocupada, seguida por restaurantes y hoteles con 9,76\%; el tercer lugar es la manufactura con $8.04 \%$ y la agricultura ocupa el cuarto lugar con 7,99\% de la población. En los demás distritos la agricultura constituye la principal actividad de la población, como se puede observar en la siguiente tabla.

Tabla 1

\section{Porcentaje de la población dedicada a la agricultura en la subcuenca}

\begin{tabular}{|l|l|}
\hline $\begin{array}{l}\text { Población dedicada a } \\
\text { la agricultura }\end{array}$ & Distrito \\
\hline Más de $80 \%$ & Huanza \\
\hline Más de $60 \%$ hasta $80 \%$ & San Mateo de Otao, San Pedro de Casta, San Juan de Iris \\
\hline Entre $30 \%$ y $60 \%$ & $\begin{array}{l}\text { Carampoma, Callahuanca, Laraos, Huachupampa, San } \\
\text { Antonio }\end{array}$ \\
\hline Menos de $10 \%$ & Santa Eulalia \\
\hline
\end{tabular}

Fuente: INEI (2017) elaborado por Guevara Floríndez (2018).

Después de la agricultura, se dedican a la construcción y actividades de transportes y comunicaciones y, en menor medida, la manufactura y la educación. Las demás actividades locales representan grupos pequeños de la población, salvo en el distrito de San Antonio, donde el 9,13\% de la PEA ocupada se dedica a actividades mineras. Debido a la búsqueda de ingresos adicionales, generalmente realizan entre una y dos actividades económicas, aunque en la zona alta de la subcuenca el promedio es mayor, hasta 1,67 por unidad agropecuaria (GRADE 2018). ${ }^{4}$

Los esfuerzos por mejores condiciones sociales parecen más de carácter individual. La articulación de las fuerzas políticas con la población es más bien débil. Por ejemplo, los planes de desarrollo local en los distritos de la subcuenca Santa Eulalia no han tenido procesos participativos

4 GRADE (2018) analiza la información del Censo Agropecuario en el periodo de 1994 a 2012 por unidades agropecuarias (UA) en las tres zonas de la subcuenca. La unidad agropecuaria es definida como el terreno o conjunto de terrenos utilizados total o parcialmente para la producción agropecuaria incluyendo el ganado, conducidos como una unidad económica. 
para su elaboración, por lo cual los que existen no reflejan la visión de los actores locales. Un estudio realizado para GWP (IDMA 2015) señala que los Planes de Desarrollo Concertado (PDC) del Gobierno Regional de Lima, la Municipalidad Provincial de Huarochirí y las Municipalidades Distritales de Callahuanca, Carampoma, Huachupampa, Huanza, Laraos, San Antonio y Casta, aprobados entre los años 2008 y 2010 por un horizonte de tiempo de entre cinco y diez años, carecieron de mecanismos de participación.

A pesar de la aprobación de los planes, dicho estudio pone de relieve una serie de omisiones en la elaboración e implementación de los planes, entre las cuales destacan que los planes de los distritos de la subcuenca no se alinean con los de los Gobiernos regional y provincial, lo que dificulta su implementación; y que los planes no fueron impresos ni socializados, son desconocidos por la población y ajenos a las organizaciones de base y espacios de concertación (a excepción de San Antonio, Callahuanca, la Provincia de Huarochirí y el Gobierno Regional de Lima).

\section{Usuarios de agua en la subcuenca y más allá de ella}

Como hemos visto, la subcuenca tiene marcadas diferencias sociales con la ciudad de Lima, acentuándose esto mientras se acerca a la parte más alta de la cuenca. Además, no cuenta con planes de desarrollo que articulen las necesidades e intereses de la población. Veamos cómo es en relación al agua.

Un informe de GRADE (2018) indica que ha habido cambios en las fuentes de agua para riego. En la cuenca baja, en 1994 las unidades agropecuarias (UA) usaban agua de ríos y manantiales, mientras en 2012 usaban además de reservorios. En la cuenca media, en 1994 usaban principalmente agua de ríos y lagos, mientras que para 2012 aumenta el porcentaje de uso de manantiales y reservorios. En la parte alta, en 1994 usaban exclusivamente agua de los ríos, mientras que en 2012 utilizan casi en la misma medida agua de los lagos.

No se explica la razón del cambio de fuentes de agua, aunque es de suponer que se ha ido incrementando la competencia por el agua de ríos y eso ha incrementado la presión sobre fuentes locales. Senamhi y Sedapal (2016: 30) indican que en la actualidad los cuerpos de agua naturales podrían considerarse como fuentes relativamente inexistentes en la subcuenca debido a la demanda creciente en la zona. Por su parte, debido a la creciente demanda de agua en la ciudad, desde hace años los cuerpos de agua se regularon artificialmente, ${ }^{5}$

5 Se represaron 15 lagunas en la parte alta, con un volumen total de 77 MMC (algunas construidas entre fines del siglo XIX y comienzos del siglo XX). Están también los trasvases y regulaciones del sistema Marcapomacocha con 157,05 MMC. 
mediante trasvases que traen gran parte del agua de la subcuenca desde la cuenca del río Mantaro, en la región hidrográfica del Atlántico, con la finalidad de que sea utilizada por usuarios fuera de la subcuenca. ${ }^{6}$

De hecho, la regulación no necesariamente favorece a todos los actores en la misma medida, pues los proyectos de trasvases y represas han sido diseñados para satisfacer las necesidades de usos particulares, como el energético o el poblacional (es el caso de Sedapal en Lima). Incluso si luego el agua es descargada en el mismo río Santa Eulalia, aguas abajo, puede más bien generar en ocasiones impactos negativos en otros usuarios.

Por otra parte, la percepción de escasez parece haber aumentado. Castro Salvador indica que «una de las principales preocupaciones de la población de la subcuenca es la falta de agua. En los últimos años, los pobladores señalan que las lluvias han disminuido progresivamente y sufren problemas de sequía y esto afecta sus actividades productivas» (2016: 53). Eso ha generado algunas iniciativas vinculadas al agua.

A la vez, algunas iniciativas locales apoyadas por la cooperación internacional han tenido ciertos avances en la subcuenca, logrando participación diversa de actores. Es el caso del PACyD - promovido por Global Water Partnership (GWP) Sudamérica- que tiene participación de empresas de energía y de Sedapal, así como de algunas comunidades y autoridades. Algo sui generis - a nivel nacional- de este caso ha sido el reconocimiento del comité coordinador del PACyD (promovido por GWP Sudamérica) por parte de la Autoridad Nacional del Agua (ANA) como grupo de trabajo especializado de trabajo, encargándole la elaboración y propuesta de la estrategia para la implementación de la gestión integrada de recursos hídricos en la subcuenca del río Santa Eulalia. ${ }^{7}$ Además, estableció que la información que genere dicho grupo será valorada para la formulación del PGRHC de CHIRILU. Aunque aún resta ver la efectividad de este aporte en la planificación de la ANA y el CRHC, es un caso atípico donde la ANA ha promovido la participación a un nivel menor de cuenca. ${ }^{8}$

De otro lado, las organizaciones de usuarios están desarticuladas, concretamente los Comités de Regantes de Santa Eulalia ${ }^{9}$ y la Junta de

\footnotetext{
6 La cuenca del río Mantaro está en la región hidrográfica del Atlántico. Es decir, al otro lado de la cadena montañosa de los Andes, donde las aguas transitan hacia el Este en dirección al Atlántico.

7 El grupo, que fue reconocido en 2015 mediante resolución jefatural 236-2015-ANA, está integrado por 17 entidades públicas y privadas.

8 Es un caso distinto al de los Comités de subcuenca en la Amazonía que se encuentran en sus inicios y están expresamente regulados como un caso particular.

9 Las comunidades campesinas se organizan para la gestión del agua en Comités, coordinan la captación y distribución del agua y el mantenimiento de la infraestructura
} 
Usuarios del Rímac. Los usuarios agrarios de Santa Eulalia tienen interés en formar una Junta con autonomía propia, pues no perciben que reciban servicios efectivos de parte de la Junta del Rímac, sin embargo, legalmente no ha sido posible por ser parte de un sistema hidráulico común (Guevara Floríndez 2018).

Las relaciones de las comunidades - o al menos parte de ellas- con las grandes empresas muestran problemas en la comunicación, así como reclamos no atendidos. Es el caso de la empresa Enel, que tiene infraestructura que transporta agua, teniendo algunas ventanas por las cuales entrega agua en el recorrido, como en Callahuanca, Huachupampa y en Casta (Castro Salvador 2016). Esto genera problemas con algunos agricultores en la parte baja debido a las horas de funcionamiento que ha establecido la empresa (entre las 5 a.m. y 12:00 m.) y la reducción del caudal como consecuencia de dicha actividad, situación que los comuneros esperan resolver llegando a un acuerdo con Enel (Castro Salvador 2016); y, al contrario, en ciertos momentos del año la empresa descarga grandes cantidades de agua sin coordinación previa, ocasionando numerosos impactos aguas abajo.

A la vez, perciben que Enel puede presentar oportunidades para beneficios locales, pues reconocen que hay iniciativas de la empresa para la localidad en materia de vías de comunicación, mediante el sistema de obras por impuestos, aunque no hay claridad sobre a quiénes beneficiaría estos proyectos (Guevara Floríndez 2018). Algo parecido pasa con el proyecto de Autisha de Sedapal, donde algunas comunidades tienen expectativas de que pudiese beneficiar también a la localidad, aunque no precisan cómo, a pesar de que para su ejecución se celebraría un convenio con Enel, GORE Lima, ANA, ACCNH y la Mancomunidad Santa Eulalia (Guevara Floríndez 2018).

Por su parte, Sedapal tiene el mandato legal — por las normas de saneamiento- de implementar proyectos para conservar las fuentes de agua de donde toma agua para Lima, incluida la subcuenca; eso incluye a Santa Eulalia, pero también al Mantaro. Según estimaciones (Bonifaz 2018), entre 2017 y 2022 Sedapal podría recibir alrededor de S/ 855 millones. No obstante, a la fecha, virtualmente no ha habido ejecución. Las comunidades tienen expectativas de que dichos proyectos les favorezcan asegurando el agua para sus actividades productivas y el consumo doméstico.

La otra iniciativa local de interés es la conformación de la Mancomunidad de las Municipalidades del Valle de Santa Eulalia (MMVSE) y de la Asociación de Comunidades Campesinas Nor Huarochirí (ACCNH), la cual 
está integrada por quince comunidades campesinas, ${ }^{10}$ además de productores agrarios y regantes, con el fin de promover proyectos de desarrollo de interés común, incluyendo temas de agua. Además, la Asociación busca una alianza para fortalecer su acceso a recursos públicos de nivel nacional y regional.

No obstante, los intereses de las comunidades tampoco son idénticos. Por el contrario, hay desacuerdos entre algunas comunidades de la subcuenca respecto de la delimitación de las tierras y sobre el desarrollo de un proyecto para la época de estiaje ${ }^{11}$ que consiste en la derivación de aguas mediante tuberías para favorecer a unas comunidades, pero que tendrían que pasar por tierras de otras (Guevara Floríndez 2018). ${ }^{12}$ Además, Castro Salvador (2016) señala la existencia de problemas de superposición de límites de tierras de las comunidades campesinas y de los Gobiernos locales, pues los límites no coinciden entre ellos.

Las tradiciones tampoco son iguales en las comunidades. Algunas conservan celebraciones comunitarias y a veces rituales, como las champerías, las faenas comunales, para la marca del ganado, mientras que en otras han caído en desuso. De hecho, no se encontraron referencias sobre tradiciones de la subcuenca (como unidad). De los testimonios existentes, las celebraciones tienen carácter más bien local (comunal), de modo que algunos ritos tienen mayor arraigo en unas comunidades y menos en otras (Guevara Floríndez 2018).

En general, la articulación entre las poblaciones locales mismas en función a proyectos de desarrollo común es, por decir lo menos, débil. Y, como se mencionó en la sección anterior, los planes de desarrollo municipales tampoco las involucran, además de no tener carácter vinculante para otros instrumentos de gestión, como planes de desarrollo nacional o departamental, por lo que su incumplimiento no genera responsabilidad, constituyéndose básicamente en documentos declarativos.

La misma normativa sobre gestión de recursos hídricos señala que la gestión del agua debe articularse con la planificación sobre el desarrollo del territorio, pero, como vimos, las poblaciones locales están en gran parte a

${ }_{10}$ La integran las comunidades de Santa Eulalia, Chaclla, Callahuanca, Mitma-Carampoma, Santiago-Carampoma, Huachupampa, Huanza, San Juan de Iris, Laraos, San Pedro de Casta, Acobamba, Quilcamachay Vicas, Collata, Jicamarca y San Mateo de Otao, aunque solo diez de ellas están en la subcuenca.

${ }^{11}$ El Proyecto Cochavijila proveería a cuatro comunidades de la margen derecha del río, mientras el Proyecto Chicoc favorecería a cinco comunidades de la margen izquierda, que tienen agua únicamente medio tiempo del año.

${ }^{12}$ Se denomina estiaje a la época del año en que no hay lluvias y las aguas de las fuentes de agua llegan al caudal mínimo. 
merced de la capacidad de los líderes locales de articular sus iniciativas con programas del Estado y, en alguna medida, de la oferta que pueda provenir de la cooperación internacional, como es el caso de la infraestructura natural, que tiene, además, recursos de Sedapal.

Desde el Estado y desde los actores que usan el agua —que están en Lima, más allá de la subcuenca-, parece asumirse que las decisiones tecnocráticas, como la planificación de los recursos hídricos, los planes de desarrollo o las actividades de los programas de gobierno, llevarán inercialmente al desarrollo de los actores locales.

La respuesta estatal ante este tipo de des-articulaciones desde la Ley de Recursos Hídricos de 2009, es la promoción de Consejos de Recursos Hídricos de Cuenca (CRHC) como los espacios institucionales que deben promover la gobernanza del agua, articulando a los distintos niveles de gobierno y a los usuarios de las cuencas. La subcuenca Santa Eulalia es parte del ámbito del Consejo de Recursos Hídricos de Cuenca Chillón - Rímac - Lurín (CRHC CHIRILU), creado en 2016, ${ }^{13}$ que tiene entre sus principales funciones participar en la elaboración del Plan de Gestión de Recursos Hídricos en la Cuenca (PGRHC) para resolver los problemas vinculados al agua y lograr el aprovechamiento sostenible del recurso.

El problema con esto es que normalmente los Consejos no tienen representantes a niveles menores (locales) de cuenca. Es el caso de la subcuenca Santa Eulalia que no cuenta con representantes locales ante el CRHC. ${ }^{14}$ Evidentemente, en ese contexto es difícil que el plan de gestión que elabore la ANA con el CRHC CHIRILU priorice los problemas de agua de las poblaciones locales.

\section{La GIRH y sus límites}

La gestión integrada de los recursos hídricos (GIRH) ha venido a ocupar una posición hegemónica a nivel internacional sobre cómo hacer gestión del agua abarcando la multiplicidad de retos que le son inherentes. La definición de uso más común es la de GWP-TAC y se entiende como el «proceso que promueve la gestión y desarrollo coordinado del agua, la tierra y los recursos relacionados, con el fin de maximizar el bienestar social y económico

\footnotetext{
${ }^{13}$ Los Consejos de Recursos Hídricos de Cuenca son formalmente órganos de la ANA creados a iniciativa de los Gobiernos regionales para promover la participación de las autoridades nacional, regionales, municipales, distintos tipos de usuarios y organizaciones de la sociedad civil.

${ }^{14}$ Ver al respecto la conformación del CRHC CHIRILU en el Observatorio del Agua (ANA s.f.).
} 
resultante, pero de manera equitativa, y sin comprometer la sostenibilidad de los ecosistemas» (2000: 22) ${ }^{15}$ Aunque dicha definición no está exenta de ambigüedad, ni responde plenamente al cómo implementarla.

La OCDE, que también postula la GIRH como uno de los pilares para el desarrollo de los países, indica que «ha dado resultados desiguales entre países y dentro de estos, y requiere de marcos operativos que consideren los aspectos a corto, mediano y largo plazo de una manera coherente y sostenible» (OCDE 2015).

Hay críticos de la GIRH por diversas razones: por ser adoptada con el discurso de sostenibilidad, sin valorar críticamente los paradigmas de la industrialización y la modernización (Abitbol 2009); por servir a una visión estatal que en la práctica desintegra la gestión del agua a nivel local y no reconoce autonomía a los actores reales de la gestión del agua (Guevara Gil 2014); por debilitar sistemas de agua que funcionan, postergando las agendas de reformas pendientes e incluso sirviendo como fachada para esconder otras agendas (Giordano \& Shah 2014). Por su parte, GWP precisa que la GIRH no es una receta, sino que aspira a proveer una dirección y unos principios a fin de que, partiendo de los objetivos de desarrollo, se promueva una coordinación multiactor.

Dourojeanni y Jouravlev critican que cuando se quiere implementar la gestión integrada por cuencas:

[...] parece asumirse que no existe ninguna cultura, ni política, ni conciencia en materia degestión delagua, en elmomento de formular la propuesta, y que solo se necesita crear una política, una cultura y una conciencia para salir adelante [...]. [Por eso,] gran parte de los llamados procesos de «culturización»y de «concientización» por el agua se vinculan a enfoques prestablecidos en otros lugares y bajo condiciones diferentes o enfoques parciales, Así, por ejemplo, se pretende «culturizar económicamente» a una población para que tome decisiones en función exclusiva de precios de mercado, para que responda a «instrumentos económicos» o análisis beneficiocosto (2001: 8).

Por ello, critican la hipótesis de que con información adecuada las personas tomarán decisiones racionales. En efecto, frecuentemente los

\footnotetext{
15 Traducido del original en inglés: «IWRM is a process which promotes the co-ordinated development and management of water, land and related resources, in order to maximize the resultant economic and social welfare in an equitable manner without compromising the sustainability of vital ecosystems».
} 
desastres ocurren porque la población ignora advertencias del peligro de ubicarse en zonas de inundación. 0 , en lugares donde todos conocen que la fuente de agua es sobreexplotada, los usuarios no se ponen de acuerdo ni modifican su comportamiento a fin de evitar el agotamiento del recurso. Hay que tomar en cuenta otros factores sociales, culturales y políticos, distintos a los técnicos.

Merrey (2008) critica la GIRH por vaga y confusa como herramienta de trabajo, al punto de parecer retórica en ocasiones. Aunque sostiene que aspectos del concepto, como la multidimensionalidad del agua, y las interconectividades propias de la cuenca son convenientes y útiles. El autor reconoce que el enfoque previo de planificación y toma de decisiones sectorial tenía un mayor sesgo top-down y excluía en general cualquier valoración de los impactos ambientales, así como la diversidad de intereses y usos. Sin embargo, señala que, al considerar la cuenca como unidad de gestión para la GIRH, no se valora suficiente la dimensión política de la gestión del agua, pasando por alto procesos sociales y económicos que siguen límites hidrográficos, así como planes e intereses de autoridades locales o regionales que se mueven por sus límites político-administrativos.

Aún más, Merrey (2008) cuestiona que la propuesta de la GIRH de alcanzar muchas metas de naturaleza distinta de forma simultánea sea siquiera posible. Además, esto distrae la atención de una agenda de problemas críticos en la cuenca, lo que puede acarrear parálisis al tener una larga lista de cosas que hacer en simultáneo. Por ello, Merrey propone lo que denomina Gestión de Recursos Hídricos Oportuna (GRH Oportuna), ${ }^{16}$ que consiste justamente en priorizar las acciones clave en la cuenca, sin perder de vista el marco de los principios de la GIRH.

Para Lankford, Merrey, Cour \& Hepworth (2007), la GRH Oportuna, en contraste con la forma en que los proyectos de gobiernos y donantes han diseñado programas de GIRH amplios y comprehensivos, permite enfocarse sin demora en las acciones prioritarias a partir de los problemas identificados en terreno, antes que en principios generales. Esto resulta en programas operativos y viables, sin el gran financiamiento que comúnmente demanda el marco comprehensivo que plantea el ideal GIRH. Para los autores, hay cierto consenso entre científicos respecto a que «en amplias cuencas, las restricciones asociadas con la escala, información disponible, políticas, conocimiento, logística, variabilidad e interfaces sistémicas, hace imposible procurar una GIRH ideal, como la define GWP» (Lankford et al. 2007: 2). ${ }^{17}$

${ }^{16}$ Merrey usa en inglés la denominación Expedient Water Resources Management.

17 Del inglés: «This is largely understood amongst most informed scientists; in large river basins, the constraints associated with scale, data availability, policing, knowl- 
Todas estas críticas a la GIRH, en el contexto de la subcuenca Santa Eulalia, cuestionan en quémedida los CRHC recogerán los proyectos prioritarios para Santa Eulalia que aborden los principales problemas, incluidos los de las poblaciones de la subcuenca. 0 si el plan de gestión - sin representantes de la subcuenca en el CRHC CHIRILU - se podría convertir en un documento omnicomprensivo que no resuelva nada en particular. Esta preocupación es aún mayor cuando, como hemos visto, las poblaciones no están representadas ni siquiera en los planes de desarrollo de los Gobiernos locales.

\subsection{La GIRH y su vinculación al discurso del desarrollo}

Otra perspectiva interesante en la crítica de la GIRH es precisamente la de su vinculación al discurso sobre el desarrollo, el cual hunde sus raíces en el enfoque de la modernización (So 1990, Valcárcel 2007). No obstante, la crítica a la modernización no es una condena inherente a la búsqueda del crecimiento económico.

De hecho, autores del postdesarrollo, como Rist (2008), sostienen que no se trata de rechazar el éxito de todos y cada uno de los proyectos llevados a cabo bajo el ideal del desarrollo, sino más bien criticar el lugar que ha tomado dicho discurso como fe, ideología, visión del futuro del mundo y modelo explicativo. Los Estados nacionales, dice Rist, deben respetar la autodeterminación y la toma de decisiones por parte de las poblaciones locales a quienes atañe la definición y realización de sus objetivos (2008: 259).

Valcárcel (2007) — citando a Jean Philippe Peemans - hace un recuento de cuatro características comunes del enfoque de la modernización en diversos autores: i) el universalismo de un modelo único de desarrollo que divide los países en desarrollados y subdesarrollados; ii) el etnocentrismo, que considera superior al desarrollo occidental; iii) el dicotomismo, que defiende la existencia de dos sectores en las sociedades: moderno y tradicional (carente de modernidad); y iv) el evolucionismo, que sostiene la necesidad de ir de forma lineal del estadio tradicional (primitivo) al moderno (superior).

En la economía, Rostow (1956) postuló que el crecimiento se da desde la sociedad tradicional hasta una de consumo masivo; desde un punto de vista sociológico, Levy (1965) indicó que, a diferencia de las sociedades no modernas, las modernas tienen normas basadas en la racionalidad y un alto grado de centralización, así como circulación de dinero y existencia del mercado. Coleman (1965) indicó que la modernización ocurre por

edge, logistics, variability and systemic interfaces invalidates the pursuit of a complete 'integrated water resources management' as defined by the Global Water Partnership» (traducción del autor). 
la adquisición progresiva de capacidad política del sistema, mediante la mayor especialización e interdependencia de las instituciones políticas y la secularización de la cultura, expresada en la igualdad ante la ley y el Estado.

$\mathrm{Al}$ respecto, el postdesarrollo ha señalado la relación entre los fenómenos de la globalización y el incremento de conflictos culturales bajo el concepto único de desarrollo. En el ámbito de la subcuenca, tanto las dinámicas migratorias que ocurrieron desde el siglo anterior hacia Lima, así como en el caso del agua, la percepción de la subcuenca como un recurso para la ciudad, dan una señal de esta relación entre lo moderno valioso y lo tradicional sin valor. No tiene que formar parte de un discurso oficial. La falta de participación de las comunidades en los planes de desarrollo y en el CRHC, además de la falta de articulación con programas nacionales que ayuden a resolver sus necesidades, parece mostrar esta dinámica subyacente.

\subsection{El desarrollo y la relación entre actores desde los aportes de los estudios sobre la paz}

En ese orden de ideas, los estudios sobre la paz pueden dar luces sobre los aspectos culturales en la relación de los actores, que pueden originar una parte de los conflictos o incluso legitimar formas de violencia.

Las comunidades (rurales) y las empresas en la Subcuenca tienen visiones distintas desde las que se relacionan con su entorno, con el agua y con la idea misma de desarrollo. El Estado no es la excepción, a través de la interpretación oficial del derecho y, desde éste, del ambiente y del agua como recursos. Desde ese punto de vista, estos estudios propondrían que las relaciones entre actores puedan reconocer el valor de visiones culturales distintas y su potencial para coexistir sin excluirse mutuamente.

Al respecto, es interesante el trabajo de Dietrich (2014), quien hace un recuento de cómo, históricamente, las sociedades cambiaron siendo pequeñas comunidades, imperios, luego los modernos Estados-nación, hasta un mundo global donde los Estados mismos se han transformado. En concordancia, habla de 5 familias (grupos) de paz(ces), según las visiones y culturas de las sociedades: energética, moral, moderna, posmoderna y transracional.

Dietrich (2006) señala que las sociedades que perciben la paz como una energía que se experimenta busca el balance entre lo social, lo natural y lo sobrenatural. Recuerda que en el antiguo Mediterráneo la relación de las comunidades con la naturaleza era representada por una divinidad femenina, maternal, de quien recibían la vida y el sostenimiento y que reflejaba la armonía. Aunque en un contexto distinto, muchas de las culturas en Perú se nutren de tradiciones donde la tierra fue valorada como madre y el agua como un elemento familiar. 
Dietrich (2006: 30) señala que, luego, cuando Roma se consolidó como imperio, Pax -antes diosa de fertilidad- pasó a ser venerada junto con la Victoria, diosa del triunfo en combate, y la paz pasa a asociarse como un período entre guerras, fruto de la victoria. Estas sociedades tenían una comprensión moral de la paz, por la cual Dios deja de ser la paz para ser quien $d a$ la paz. De igual modo, para el autor, en las grandes religiones, como el cristianismo o el islam, la paz es una recompensa de Dios por un comportamiento moral. Esto va de la mano con la formación de sociedades más complejas y con mayores estructuras que separan las actividades (incluso religiosas) por especialización. En ellas, revaloran lo divino masculino asociado al orden y a la paz.

La modernidad concibió la paz basada en la razón, en el paradigma de que la ciencia era garante de la verdad. Estas sociedades afianzan el Estado y el mercado como ideales políticos de la vida en grupo y de mediación de la razón (Dietrich 2006). En esta lógica, los problemas sociales pueden ser entendidos y resueltos y, por ende, la paz y el progreso pueden ser instaurados.

Con la posmodernidad y la crítica a los límites de la razón, en el campo de estudios sobre la paz se revisaron críticamente las ideas de paz y progreso, pues un significado unívoco ha servido frecuentemente, como justificación para modelos de dominación autoritarios. Además, la consciencia sobre los límites de la razón abrió las puertas para aceptar la pluralidad de verdades (y de paces) que permiten la coexistencia de las diferencias.

Para Dietrich (2006), por úlitmo, la paz transracional no pretende rechazar la racionalidad, sino quitarle la pretensión de absoluto, integrando (reconciliando) la razón con elementos energéticos presentes en otras visiones y experiencias de la paz $\mathrm{u}$ otras formas de acercarse a las verdades. Enfoques como la psicología transpersonal recuperan el sentido trascendente (más allá del individuo) y transpersonal (en relación con otros), sin el cual «los seres humanos se tornan enfermos, violentos, nihilistas, desesperados y anómicos. Necesitan algo más grande que ellos mismos para sentir reverencia en forma natural y empírica, más allá de los límites establecidos por Iglesias e instituciones» (2006: 39). ${ }^{18}$

Esta tensión de visiones parece presente en la subcuenca Santa Eulalia entre los intereses de actores de Lima, para quienes el agua es meramente un recurso, y las poblaciones locales, rurales, donde el agua forma parte de un horizonte más amplio de vida. Entre los que usan el agua como un servicio esencial que provee Sedapal y aquellos para quienes el agua involucra otros valores, como tradiciones antiguas, un ecosistema que los sostiene, así como sus actividades económicas de subsistencia. 
El paradigma del desarrollo de la GIRH y la forma como se busca implementar en el CRHC CHIRILU desde el Estado, no ha dado cabida a la visión de las poblaciones locales sobre el agua o el desarrollo. De momento no hay incentivos suficientes para que los diversos actores se sienten en una misma mesa con ese propósito, a pesar de las iniciativas locales como el PACyD y la ACCNH. La generación de espacios donde puedan integrar las distintas visiones y necesidades podría abrir un camino a la construcción de lo que Dietrich llama una paz transracional.

\section{Reflexiones finales}

A pesar de las iniciativas locales, los actores de la subcuenca no han podido concretar una participación que les permita articular sus necesidades con recursos y decisiones de niveles de gobierno mayores a nivel de los planes de desarrollo locales y nacionales.

Las distintas visiones y marcos culturales de los actores de la subcuenca - por ejemplo, las comunidades y las empresas-, más allá de relaciones asistenciales, parecen tener dificultad en encontrar puntos en común. Entre otras cosas, Santa Eulalia es interesante por su relevancia para actores fuera de ella, nada menos que de la capital del país. Convendría realizar mayores investigaciones que exploren en qué medida diferentes concepciones del desarrollo y de la paz podrían integrarse en un marco de referencia común. La falta de espacios formales para ello es un problema.

El CRHC que debiera ser el espacio que acerque a los usuarios y las autoridades en torno al agua, no incluye representantes locales. En ese sentido, la subcuenca Santa Eulalia muestra que el enfoque de la GIRH, como se viene implementando en el Perú, frecuentemente no integra las visiones y expectativas locales. No obstante, esta brecha de articulación entre lo regional y lo local no es exclusiva de estos actores. A nivel local, el concepto de cuenca no necesariamente encuentra correlato en la existencia de una visión común entre las comunidades, o de mecanismos de coordinación entre ellos, deviniendo en alguna medida en una imposición teórica. Es también el caso de los Comités de Usuarios y la Junta de Usuarios del Rímac, donde la relación se percibe más como una imposición.

El enfoque mismo de GIRH, a la luz de la experiencia de Santa Eulalia, debiera ser materia de una discusión crítica para evaluar sus alcances y sus límites, pues, aunque promueve principios generales positivos, su implementación presenta problemas no pequeños.

Esto está estrechamente ligado a la discusión sobre el desarrollo local, toda vez que, por un sesgo cultural, los gobiernos de distintos niveles y los 
REVISIÓN CRÍTICA DE LOS ALCANCES Y LÍMITES DE LA GESTIÓN INTEGRADA DE RECURSOS HÍDRICOS (GIRH): El CASo de la Subcuenca Santa Eulalia / Víctor Guevara

actores exógenos parecen ver el desarrollo como un proceso tecnocrático, prescindiendo de dimensiones locales, culturales y políticas.

La mirada desde las muchas paces (Dietrich 2006) invita a valorar de qué manera el integrar las diferentes experiencias sobre el mundo — digamos, las de las empresas, el Estado y las comunidades - puede ser determinante en la construcción de una paz que dé un lugar a las diferencias en una gestión del agua realmente integradora.

\section{Referencias}

AвітвоL, E. (2009). Developing Water and Marginalising Israel/Palestinian Peace: A Critical Examination of the Red Sea-Dead Sea Canal Feasibility Study Process. Journal of Peacebuilding and Development, 5(1): 35-49.

ANA (Autoridad Nacional del Agua) (s.f.). Consejo de Recursos Hídricos de Cuenca Interregional Chillón-Rímac-Lurín - CRHC CHIRILU. En Observatorio del Agua. Ana: http://observatoriochirilu.ana.gob.pe/acciones-derespuesta/avances-institucionales/CRHC-chirilu

Bonifaz, J.L. (27 de junio de 2018). Tarifas de agua para el Rímac. Obtenido de El Comercio. https://elcomercio.pe/economia/opinion/tarifas-aguarimac-jose-luis-bonifaz-noticia-453094

Castro Salvador, S. (2016). Gestión del Agua y la Descentralización. El Caso del Río Santa Eulalia en la Periferia de la Metrópoli de Lima (Perú). Memoria del Master de investigación Países emergentes y en desarrollo. Lima: Université Paris 1. Panthéon Sorbonne.

Coleman, J. S. (1965). Education and Political Development. New Jersey: Princeton University Press.

Dietrich, W. (2006). Peaces: An Aestetic Concept, a Moral Need or a Transrational Virtue? *asteriskos, Journal of International and Peace Studies, (1/2): 25-45.

Dietrich, W. (2014). A Brief Introduction to Transrational Peace Research and Elicitive Conflict Transformation. Journal of Conflictology, 5(2): 48-57.

Dourojeanni, A., \& Jouravlev, A. (2001). Crisis de gobernabilidad en la gestión del agua. Santiago de Chile: Naciones Unidas - CEPAL.

García Rivero, A. E. (2017). Línea Base de la Subcuenca Santa Eulalia. Lima: GWP.

Giordano, M., \& SHAH, T. (2014). From IWRM back to integrated water resources management. International Journal of Water Resources Development, 30(3): 364-376. 
GRADE (Grupo de Análisis para el Desarrollo) (2018). Estrategia para la implementación de MERESE y desarrollo agrario en la sub cuenca de Santa Eulalia. Informe Final. Lima: GRADE.

Guevara Floríndez, V. (2018). El caso de la Subcuenca Santa Eulalia: Entre la gobernanza del agua y el llamado a las muchas paces. Tesis para la Maestría en Paz, Desarrollo, Seguridad y Transformación de Conflictos Internacionales de la Universidad de Innsbruck. http://diglib.uibk.ac.at/ ulbtirolhs/content/titleinfo/3006180

Guevara GiL, A. (2014). La gestión (des)integrada del agua en la cuenca alta del río Ica: el caso de Santa Rosa de Tambo, Huancavelica. En G. Damonte, \& M. T. Oré. ¿Escasez del agua? Retos para la gestión de la cuenca del río Ica (pp. 173-226). Lima: Fondo Editorial de la Pontificia Universidad Católica del Perú.

GWP-TAC (2000). Integrated Water Resources Management. Stockholm: Global Water Partnership.

GWP (2017). Reporte de Daños del Fenómeno del Niño-FEN, 2017. Lima.

IDMA (2015). Revisión de los Planes de Desarrollo Concertado de la Sub-Cuenca Santa Eulalia. Instituto de Desarrollo y Medio Ambiente. Lima: GWP.

Instituto NaCional DE Estadística E Informática - INEI. Censo Nacional 2017. Recuperado el 15 de abril de 2018. https://www.inei.gob.pe/ estadisticas/censos/

Instituto de Planificación de Paisaje y Ecología (2014). Estrategia de Infraestructura Ecológica de Lima. Stuttgart: Instituto de Planificación de Paisaje y Ecología.

Lankford, B. A., Merrey, D. J., Cour, J., \& Hepworth, N. (2007). From Integrated to Expedient: An Adaptive Framework for River Basin Management in Developing Countries. Colombo: International Water Management Institute.

Levy, M. J. (1965). Patterns (Structures) of Modernization and Political Development. The Annals of the American Academy of Political and Social Science, 358(1): 29-40.

Matos MaR, J. (1984). Desborde Popular y Crisis del Estado. Lima: CONCYTEC.

MERREY, D. J. (2008). Is normative integrated water resources management implementable? Charting a practical course with lessons from Southern Africa. Physics and Chemistry of the Earth (33): 899-905. 
REVISIÓN CRÍTICA DE LOS ALCANCES Y LÍMITES DE LA GESTIÓN INTEGRADA DE RECURSOS HÍDRICOS (GIRH): EL CASO DE LA Subcuenca SANTA Eulalia / Víctor GuEVARA

OCDE (2015). Principios de Gobernanza del Agua de la OCDE. OECD Better: Policies for Better Lives. https://www.oecd.org/cfe/regional-policy/OECDPrinciples-Water-spanish.pdf

Programa de Naciones Unidas Para el Desarrollo. Indice de Desarrollo Humano. En https://www.pe.undp.org/ Consultada el 28 de mayo del 2018.

Rist, G. (2008). The History of Development. From western origin to global faith. London \& New York: Zed Books Ltd.

SENAmhi \& Sedapal (2016). Estudio de vulnerabilidad climática de los recursos hidrícos en las cuencas de los ríos Chillón, Rímac, Lurín y parte alta del Mantaro (resumen ejecutivo). Lima: SENAMHI.

So, A. Y. (1990). Social Change and Development: Modernization, Dependency and World-System Theories. California: Sage Publication.

VALCÁRCEL, M. (2007). Génesis y Evolución del concepto y enfoques sobre el desarrollo. Departamento de Ciencias Sociales-PUCP. http://cisepa.pucp. edu.pe/wp-content/uploads/2016/07/Desarrollo-y-Desarrollo-Rural_ Enfoques-y-reflexiones_Marcel-Valcárcel.pdf 